\title{
Age-Related Differences in
}

Interhemispheric Visuomotor Integration Measured by the Redundant Target

\section{Effect}

\section{Linnet, E}

http://hdl.handle.net/10026.1/14635

10.1037/a0024905

PSYCHOLOGY AND AGING

American Psychological Association (APA)

All content in PEARL is protected by copyright law. Author manuscripts are made available in accordance with publisher policies. Please cite only the published version using the details provided on the item record or document. In the absence of an open licence (e.g. Creative Commons), permissions for further reuse of content should be sought from the publisher or author. 


\title{
Age-Related Differences in Interhemispheric Visuomotor Integration Measured by the Redundant Target Effect
}

\author{
Elisabeth Linnet and Matthew E. Roser \\ University of Plymouth
}

\begin{abstract}
Presentation of bilateral redundant visual stimuli produces faster reaction times (RT) than presentation of a single unilateral stimulus; an effect known as the redundant target effect (RTE; Miller, 1982), and is a means of testing interhemispheric visuomotor integration (Ouimet, 2009). RTEs that exceed expectations, based on Miller's race model of inequality (RMI), are referred to as "enhanced RTEs" and imply neural coactivation. Paradoxically, enhanced RTEs are observed in cases of corpus callosum disruption. The Hemispheric Coactivation Hypothesis accounts for this paradox by positing that bihemispheric processing occurs to both unilateral and bilateral stimuli in the normal brain, but occurs only with bilateral stimuli in the disconnected brain. Neuroimaging has revealed decreases in the microstructural integrity of the corpus callosum with age (Ota et al., 2006), but research investigating the bilateral RTE in healthy older individuals is lacking. The present study investigated the bilateral RTE in healthy younger and healthy older adults using simple RT and choice RT tasks. Our prediction that older individuals would show significantly larger RTEs than younger individuals was found to be true for both tasks. Tests of the RMI produced little evidence for coactivation. The crossed-uncrossed difference, generally used as a means of testing visuomotor interhemispheric transfer, was also investigated, but no age effects were found. The observation of greater RTE in age is congruent with the Hemispheric Coactivation hypothesis (Miller, 2004) in which callosal disconnection is associated with increased RTE.
\end{abstract}

Keywords: redundant target effect, redundancy gain, aging, visuomotor, corpus callosum

Sensorimotor integration is of fundamental importance in interactions with the world, and has been shown to be adversely affected by age (Reuter-Lorenz \& Stanczak, 2000). When participants are required to make speeded unimanual responses to visual stimuli, the presentation of bilateral redundant visual stimuli usually produce faster reaction times than does a single unilateral stimulus; an effect which is known as the redundant target effect (RTE) or redundancy gain (Miller, 1982). By presenting visual stimuli in both visual hemifields simultaneously, each target activates the contralateral hemisphere, thereby allowing investigation of visuomotor interhemispheric integration (Ouimet et al., 2009).

A phenomenon known as the crossed-uncrossed difference (CUD), indexing the difference in reaction time (RT) between uncrossed responses (stimuli in the visual hemifield ipsilateral to the responding hand) versus crossed responses (stimuli in the visual hemifield contralateral to the responding hand), has been used as a measure of callosal function and visuomotor interhemispheric transfer (Reuter-Lorenz \& Mikels, 2005; Schulte, Sullivan, Müller-Oehring, Adalsteinsson, \& Pfefferbaum, 2005). The finding that patients who have had partial or complete section of the corpus callosum (callosotomies) tend to produce much larger RTEs and CUDs than neurologically intact individuals suggests

This article was published Online First August 15, 2011

Elisabeth Linnet and Matthew E. Roser, School of Psychology, University of Plymouth, Devon, United Kingdom.

Correspondence concerning this article should be addressed to Elisabeth Linnet, School of Psychology, University of Plymouth, PL4 8AA, Devon, United Kingdom. E-mail: elisabeth.linnet@plymouth.ac.uk that the corpus callosum plays a significant role in mediating interhemispheric transfer (Corballis, Corballis, \& Fabri, 2003). The corpus callosum, the largest cerebral commisure in humans, allows interaction and transfer of information between the two cerebral hemispheres, and is thought to play an essential role in the integration of lateralized sensory, motor and cognitive processes (Reuter-Lorenz \& Stanczak, 2000).

Evidence from neuroimaging studies has revealed age-related decreases in the structural integrity of the corpus callosum (Ota et al., 2006), and there is evidence to suggest that disruption of white matter integrity are associated with similar declines in interhemispheric transfer (Sullivan \& Pfefferbaum, 2006). Concomitantly, behavioral evidence suggests that bihemispheric processing may be beneficial to older adults at lower levels of task complexity (Reuter-Lorenz, Stanczak, \& Miller, 1999), and neuroimaging studies reveal that older adults show greater bilateral activation than younger adults for a number of tasks (for a review, see Cabeza, 2002).

The focus of the present study was to investigate, using a simple reaction time task and a choice reaction time task, whether agerelated differences in visuomotor interhemispheric transfer and integration manifest in an increased RTE in age.

\section{The Redundant Target Effect and Coactivation Models}

The RTE is a measure of visuomotor interhemispheric integration, examining parallel processing of visual stimuli presented simultaneously to each hemisphere (Schulte et al., 2005). Numerous versions of this task have been investigated with both neuro- 
logically intact individuals and individuals with particular neuropsychological abnormalities, such as people who have had partial or complete callosotomies (Iacoboni \& Zaidel, 2003a; Roser \& Corballis, 2002, 2003).

A number of mathematical models have been proposed to account for the response facilitation by redundant targets. The majority of the research focuses on two models; (1) probability models or (2) coactivation models. Probability models, also called race models, assume that the RTE arises purely from statistical facilitation (Miller, 1982; Miller, 2004). Raab (1962) suggested a race model where each stimulus is transmitted along separate channels; each stimulus initiates a separate process which is capable of triggering the motor response, and the final response is simply determined by whichever process completes first. Hence, provided that the probability distributions for either of the unilateral stimuli overlap, the average RT in bilateral trials should be faster than the average RT of either unilateral trial (Miller \& Van Nes, 2007). The race model therefore predicts RTE based on statistical facilitation for two processes that are completely independent. Miller's (1982) seminal work described the race model inequality (RMI), which acts as an upper bound on the statistical facilitation. A RTE exceeding that predicted by the RMI (generally referred to as an enhanced RTE) is generally interpreted as RT facilitation due to underlying neural summation, or coactivation mechanisms, in which initially independent processes converge to produce a response (Colonius \& Diederich, 2006; Miller, 2004).

In neurologically intact participants, the facilitation of RT by redundant bilateral stimuli generally does not exceed that predicted by the RMI, wherefore it may be ascribed to statistical facilitation rather than coactivation (Corballis, 2002; Roser \& Corballis, 2002). There are, however, studies (Miniussi, Girelli, \& Marzi, 1998; Savazzi \& Marzi, 2002; Roser \& Corballis, 2003) that report enhanced bilateral RTEs in normal participants, supporting the coactivation model and suggesting that RTEs are not due entirely to statistical facilitation.

It has been demonstrated that split brain individuals often show unusually large RTEs, thereby violating the RMI to a far greater extent than the intermittent violations observed in normal individuals (Iacoboni \& Zaidel, 2003a; Roser \& Corballis, 2002, 2003; Reuter-Lorenz, Nozawa, Gazzaniga, and Hughes, 1995). That the RTE should be larger in split brain patients than neurologically intact individuals has been called "paradoxical" (Corballis, 1998, p. 1795), since the corpus callosum is assumed to play a crucial role in interhemispheric interaction - and interhemispheric interaction should be restricted to subcortical pathways, at least in those patients with complete section of the corpus callosum. Corballis (2002) noted that this is particularly surprising considering split brain patients usually show much larger CUDs, reflecting reduced interhemispheric communication, as well as enhanced RTEs implying enhancement of interhemispheric communication. An association of large CUD with large RTE was not apparent within a group of normal participants (Corballis, 2002), nor within a group of alcoholics (Schulte, Pfefferbaum, \& Sullivan, 2004), suggesting that disruption of the corpus callosum, such as in split-brain patients, was required for enhanced RTE, and that the CUD and the RTE may depend on different callosal channels (Corballis, 2002; Schulte et al., 2004).

Miller's (2004) hemispheric coactivation model accounts well for the paradoxical effect of enhanced RTEs as well as the exis- tence of particularly large CUDs in split brain individuals, and why these effects are usually much less pronounced in neurologically intact individuals. According to Miller's (2004) model the presentation of a lateralized stimulus activates the visual area in the contralateral hemisphere, which in return activates the motor areas of both hemispheres. The activation of each motor area then combines into a total motor activation, and a coactivated response is made once a criterion is reached. When bilateral stimuli are presented each sensory area activates both motor areas; consequently, the total motor activation is combined across hemispheres, resulting in coactivation. Thus, the assumption is that both hemispheres are involved in producing key-press responses, even with unimanual responses to uncrossed stimuli (Miller, 2004). This view has been supported by electrophysiological evidence demonstrating that unimanual responses in uncrossed conditions show initial sensory lateralization, followed by bilateral frontal, central, and occipital activations (Saron, 1999) and furthermore magnetoencephalography (MEG) studies have demonstrated that unimanual index finger movement involve bilateral motor cortex activations (Babiloni et al., 1999).

Under this schema, it is suggested that neurologically intact individuals only show a small RT advantage for bilateral stimuli because callosal connectivity results in greater bihemispheric processing of unilateral stimuli than in patients, which is reflected in little difference in brain activation patterns between unilateral and bilateral stimulus input (Miller, 2004; Roser \& Corballis, 2003). In split brain individuals, however, a unilateral stimulus does only activate the contralateral hemisphere due to significantly reduced interhemispheric communication. The hemisphere not receiving the unilateral input may be activated via subcortical pathways, but these are too slow to significantly influence responses in a simple RT task (Roser \& Corballis, 2002). Thus, in split brain patients, the unstimulated hemisphere does not contribute to the response activation (or is, as a minimum, substantially delayed), whereas bilateral stimuli activate both hemispheres directly, thereby resulting in substantially faster responses and an enhanced RTE (Roser \& Corballis, 2002; Miller, 2004).

The limited research investigating the effects of intrahemispheric redundant presentation provides further support for the importance of the corpus callosum in producing enhanced RTEs; both neurologically intact individuals as well as split brain patients tend to produce relatively small RTEs in intrahemispheric conditions and interhemispheric presentation appears to be necessary for an enhanced RTE to occur (Iacoboni, Ptito, Weekes, \& Zaidel, 2000; Reuter-Lorenz et al., 1995; Ouimet et al., 2009).

\section{The Crossed-Uncrossed Difference}

The CUD is generally used as a means of testing visuomotor interhemispheric transfer, and there is evidence to suggest that age-related declines in white matter integrity are associated with similar declines in interhemispheric transfer (as measured by the CUD; Reuter-Lorenz \& Stanczak, 2000; Schulte et al., 2004; Schulte et al., 2005). As each visual field projects to the contralateral hemisphere and distal effectors are controlled almost exclusively by the contralateral hemisphere, the basic tenet of the CUD paradigm is that crossed conditions (e.g., right hand/ left visual field) requiring interhemispheric relay via the cerebral commisures 
should yield longer RTs than uncrossed conditions (e.g., right hand/right visual field; Zaidel \& Iacoboni, 2003).

The role of the corpus callosum in mediating the CUD has gained support from the findings that the CUD increases dramatically, often to around $70 \mathrm{~ms}$, in patients who have had partial or complete callosotomies and those with callosal agenesis, although there are considerable individual differences (Corballis et al., 2003; Roser \& Corballis, 2002; Roser \& Corballis, 2003). Previous research investigating the CUD in healthy older adults have reported equivocal results; some studies have demonstrated significant increases in the magnitude of the CUD compared to younger adults (Jeeves \& Moes, 1996; Reuter-Lorenz \& Stanczak, 2000; Schulte et al., 2004; Schulte et al., 2005), while others have found no such effect (Hoptman, Davidson, Gudmundsson, Schreiber, \& Ershler, 1996). The validity of the CUD as an index of callosal transmission time has been called into question by studies in which only approximately between half to two thirds of participants showed positive CUDs, suggesting that the simple anatomical model on which the CUD is based may be invalid (Saron \& Davidson, 1989; Davidson, Leslie, \& Saron, 1990).

\section{Age-Related Changes in Neurophysiology and Patterns of Functional Activation}

Callosal disruption, such as agenesis or disconnection, is associated with enhanced bilateral RTEs, suggesting that the corpus callosum plays a significant role in the bilateral RTE (Roser \& Corballis, 2002; Roser \& Corballis, 2003; Roser, et al., in press; Schulte et al., 2006; Ouimet et al., 2009). Accumulating evidence suggests that callosal degeneration occurs with normal ageing, raising the possibility that the RTE will show a corresponding age-related increase. Studies using volumetric magnetic resonance imaging (MRI) have demonstrated significant reductions in the size of the corpus callosum with age (Good et al., 2001; Raz et al., 1997; Raz et al., 2005; Sullivan, Pfefferbaum, Adalsteinsson, Swan, \& Carmelli, 2002). Studies using diffusion tensor imaging (DTI), which allows investigation of the microstructural integrity of white matter (see Chanraud, Zahr, Sullivan, \& Pfefferbaum, 2010), have suggested that the structural integrity of white-matter tracts declines with age (Sullivan \& Pfefferbaum, 2006). Furthermore, age-related loss of white-matter volume (Raz \& Rodrigue, 2006; Sullivan, Rohlfing, \& Pfefferbaum, 2010), and degeneration of callosal microstructure (Cámara, Bodammer, RodriguesFornells, \& Tempelmann, 2007; Head et al., 2004; Salat et al., 2005; Bennett, Madden, Vaidya, Howard, \& Howard, 2010; Burzynska et al., 2010; Cámara et al., 2007; Ota et al., 2006; Salat et al., 2005; Sullivan \& Pfefferbaum, 2006; Sullivan et al., 2010) is particularly marked in anterior regions of the brain.

Despite the evidence suggesting a reduction of white matter integrity, there is ample evidence from neuroimaging studies suggesting that older adults show greater bilateral activation for a number of both higher order cognitive processes as well as simple sensory and motor tasks that evoke unilateral activation in younger individuals (for reviews, see Cabeza, 2002; Park \& Reuter-Lorenz, 2009). During the execution of a number of motor tasks, older adults tend to show stronger activations in the same areas that are also activated in younger adults, as well as recruitment of additional brain regions not observed in younger adults (Carp, Park, Polk, \& Park, 2010; Heuninckx, Wenderoth, \& Swinnen, 2008;
Seidler et al., 2010). Although the increased bilateral activations observed in older adults may seem paradoxical in light of the reduction of white matter integrity, there is evidence to suggest that increased bilaterality is associated with enhanced performance of both cognitive tasks (Cabeza, Anderson, Locantore, \& McIntosh, 2002) and motor tasks (Mattay et al., 2002; Heuninckx et al., 2008), which suggests that increased bilateral activations may counteract neurocognitive decline.

The neuroimaging evidence has been supported by behavioral studies. Banich (1998) demonstrated that as task difficulty increases, younger adults engage in increasingly bilateral processing. Furthermore, in a within- versus across-hemisphere matching task, Reuter-Lorenz et al. (1999) demonstrated that older adults benefitted from across-hemisphere matching at lower levels of task complexity. This led these authors to suggest that at high levels of complexity the benefits of bihemispheric processing may outweigh the costs of interhemispheric communication for both age groups, and that bihemispheric processing may become beneficial to older adults at lower levels of complexity than for younger adults.

Thus, several lines of evidence suggest that interhemispheric connectivity and functional integration are affected by age. By contrast, only a few studies have investigated the RTE in healthy older adults, with most finding greater RTE in aged participants. None of these studies, however, have investigated redundancies of stimulus input to the two hemispheres. Bucur and colleagues employed redundancies of modality (Bucur, Allen, Sanders, Ruthruff, \& Murphy, 2005) and dimension (Bucur, Madden, \& Allen, 2005) in simple speeded-response paradigms and found enhanced RTEs, implying coactiviation, in adults ranging in age from 60 to 85 years of age. RTEs were larger in aged adults than in young controls, especially under demanding task conditions, and were interpreted as suggestive of compensation for age-related deficits in perceptual processing. Allen and colleagues investigated redundancies of stimulus presentation in visual search paradigms and attributed age effects to increased perceptual noise (Allen, Madden, Groth, \& Crozier, 1992), decrements in attentional capacity (Allen, Groth, Weber, \& Madden, 1993), and decrements in stimulus identification and attentional selection (Allen, Weber, \& Madden, 1994).

In contrast to the developing literature investigating age differences in the effects of redundant dimensions or modalities, there exist no studies investigating the effect of age on processing of redundant stimuli presented separately to the two cerebral hemispheres. The present study investigates age effects on the bilateral RTE and is motivated by the considerable, and growing, literatures that describes age effects on functional asymmetry (Cabeza, 2002; Park \& Reuter-Lorenz, 2009) and interhemispheric structural connectivity (Sullivan \& Pfefferbaum, 2006; Raz \& Rodrigue, 2006), as well as previous demonstrations of enhanced RTEs in cases in whom interhemispheric callosal transfer is impaired (Roser \& Corballis, 2002; Roser \& Corballis, 2003; Roser, et al., in press; Schulte et al., 2006). The study, therefore, addresses a type of redundancy gain for which there are good reasons for expecting age effects to manifest.

\section{The Present Experiments}

The present study investigated age effects on visuomotor interhemispheric integration in healthy younger and older individuals, 
using the bilateral RTE within the visual modality in a manner similar to previous investigations in surgical and congenital cases with disruption to the corpus callosum. Both the simple reaction time task and the choice reaction time task included unilateral visual stimuli (restricting input to the contralateral hemisphere) and bilateral visual stimuli presented to both hemispheres simultaneously.

On the basis of the theoretical assumptions in Miller's (2004) coactivation account, according to which reduced callosal connectivity is associated with increased RTEs, the findings of enhanced RTEs in split brain individuals, and evidence for neurophysiological degeneration of the corpus callosum, it was predicted that (1) older individuals would exhibit larger RTEs and larger violations of the RMI than younger individuals. Following from neuroimaging evidence revealing that frontal regions including the anterior corpus callosum are particularly vulnerable to age related decline in white matter integrity, it was predicted that (2) age effects would be maximal for the choice RT task, given that it likely involves greater reliance on frontal regions involved in response selection.

The use of multiple tasks has several advantages over earlier investigations of the bilateral RTE. Beyond simply the observation of bilateral RTEs in aged and in young participants, the pattern of results arising from the use of two response tasks, such as increased age effects with increased task complexity, may be informative and increase confidence in the interpretation of age differences. The observation of greater age effects in choice RT tasks compared to simple RT tasks would be congruent with evidence (Raz \& Rodrigue, 2006; Sullivan et al., 2010) for an anteriorposterior gradient of brain structural degeneration in age. Furthermore, employing more than one paradigm will allow the generality and reliability of age effects on the bilateral RTE, beyond the specific response requirements of any single paradigm, to be determined.

A second line of investigations were related to interhemispheric visuomotor transfer, as measured by the CUD. On basis of the same assumptions listed above, it was predicted that: (3) younger individuals would show small, insignificant CUDs in both tasks and that (4) older individuals were expected to show larger CUDs, particularly in the choice RT task.

\section{Method}

\section{Participants}

Twenty-nine healthy younger adults (seven men and 22 women aged 20-30 years, $M=22$ ), and 23 healthy older adults (11 men and 12 women aged 55-74 years, $M=61$ ) participated in this study. In order to reduce the likelihood of recruiting participants with cognitive impairments, only participants who had normal or corrected-to-normal vision and had no history of neurological disorders or stroke were recruited. An additional four younger and five older adults participated but their data were excluded from all analysis due to excessive error rates (see results section). The younger participants were undergraduate psychology students from the University of Plymouth, and the older participants were recruited through the School of Psychology's participant database. All younger participants had a minimum of 12 years of education. In the group of older participants, 17 had a minimum of 12 years of education, the remaining six had between 9 and 12 years of education.

\section{Materials and Apparatus}

E-prime software (version 2.0) was used for stimulus presentation and a stimulus response box for response recording. Participants were seated comfortably in front of a 20 in. Samsung LCD monitor using a chin-rest, ensuring a viewing distance of $57 \mathrm{~cm}$. Participants' handedness was assessed using the amended short version of the Edinburgh Handedness Inventory (EHI; Oldfield, 1971). Ten items assessed hand preference for everyday tasks such as writing and using cutlery, and 2 items assessed eye and foot preferences. The Mini-Mental State Examination (MMSE; Folstein, Folstein, \& McHugh, 1975) is a short questionnaire that screens for cognitive impairment, and is widely used in studies of normal cognitive function in aged participants who are free of cognitive impairment as well as participants with mild cognitive impairment (Dickerson \& Sperling, 2008; Langan et al., 2010; Salat et al., 2005). All participants scored greater than 26 on the MMSE, indicating normal cognitive function.

\section{Stimuli and Procedure}

For the simple reaction time experiment the stimuli consisted of filled white circles, and for the choice reaction time experiment the stimuli consisted of white Xs and Ts. All stimuli subtended approximately $2.8^{\circ}$ in visual angle and the inner edges of all stimuli were presented approximately $5^{\circ}$ from a central fixation cross. In both tasks the stimuli were presented for $800 \mathrm{~ms}$ on a black background to the right, left or both sides of the fixation cross.

The simple RT task consisted of a total of 384 trials. It was divided into four blocks of 96 trials with two blocks per hand. Thus, in each block there were 30 trials in which the circles were presented in the left visual field (LVF), 30 trials in which they were presented in the right visual field (RVF) and 30 trials in both visual fields (bilateral presentations). Each block also included 6 catch trials in which no target was presented, to prevent participants from making anticipatory responses. The choice RT condition also comprised a total of 384 trials divided into four blocks. Each block consisted of 30 presentations in each visual field condition (LVF, RVF and bilateral presentations) and six catch trials. Half of the presentations in each of the visual field conditions were Xs, the other half Ts.

Each block was initiated by pressing the spacebar. A white fixation cross then appeared in the middle of the screen. Participants were instructed to maintain their gaze at the fixation point throughout the experiment. Prior to each trial the cross blinked, indicating that the stimulus was about to be presented. On each trial there was a variable interval of $500,750,1000,1250$, or 1500 ms before the stimulus appeared. Within each block, each of the five intervals was paired six times with each stimulus condition, all of which were presented randomly (including the catch trials). The target stimulus appeared on the screen for $800 \mathrm{~ms}$. As responses were allowed from stimulus onset, RTs were measured relative to the onset of the target stimulus. In the catch trials participants were required to withhold their responses for $800 \mathrm{~ms}$ - and, if unsuccessful, a warning sign appeared. Each of the two experimental conditions was preceded by a short practice trial. 
Participants were randomly allocated to begin with either the simple or choice task, and were instructed to rest their forearm on the desk in front of them in order to maximize the use of distal musculature. For the simple RT task, the response box was placed horizontally at the midline in front of participants, and they were instructed to use their left and right index fingers on the far left and right button, respectively. The positions of these buttons deviated from the midline by only approximately one inch and can be considered essentially midline. For the choice reaction time task, the box was placed vertically in front of participants in order to avoid the Simon effect, according to which performance is facilitated if the position of the hand (or in this case the fingers) is congruent with the relative position of the stimulus (Simon, 1969). When responding with their right hand, participants were instructed to press the upper button with their middle finger when an $\mathrm{X}$ was presented and to press the button second from the top with their index finger when a $\mathrm{T}$ was presented. When responding with their left hand the box was turned 180 degrees, the instructions were otherwise identical.

\section{Calculation of RTs, the CUD, and the RTE}

For calculation of RTs, the RTE, and the CUD, medians were used as the best measure of central tendency, thereby avoiding contamination by outliers. Median RTs, CUDs, and RTEs were calculated separately for each participant, each hand, and each task in the following manner. First, trials in which participants failed to respond, or in which an incorrect response was made in the choice task, were deleted. The median RTs for each stimulus presentation conditions (crossed, uncrossed, bilateral) were calculated. The CUD was then obtained by subtracting the median RT in uncrossed conditions from the median RT in crossed conditions, and the RTE was obtained by subtracting the median RT in bilateral conditions from the median RT in uncrossed conditions.

\section{Results}

\section{The Edinburgh Handedness Inventory}

Participants' handedness was calculated in accordance with the short version of the EHI, and the standard cut-off points were applied; -100 to $-40=$ left-handed, -40 to $+40=$ ambidextrous and +40 to $+100=$ right-handed (Oldfield, 1971). One participant in each age group was classified as ambidextrous, the remaining participants as right-handed. The mean handedness scores were similar for both age groups, with scores of 80.65 $(S D=20.86)$ and $76.41(S D=19.54)$ for the older and younger group, respectively.

\section{The Mini-Mental State Examination}

In the MMSE, a maximum score of 30 points can be obtained if the participant answers all items correctly (Folstein et al., 1975). All participants were classified as having normal cognitive function as per the MMSE (to meet this criteria, a minimum score of 26 is required). The mean scores for the younger participants were $M=29.31(S D=1.26)$ and older participants had a mean score of $M=29.48,(S D=0.79)$.

\section{Educational Level, Age, and Performance}

To ensure that the lower educational level among the six older adults with less than 12 years of education did not influence the data in any way, z-scores were calculated from the raw RT data. None of the six individuals had z-scores greater than 2, indicating that they did not deviate from the older group-average.

Within the older group there was no significant correlation between age and mean RTs $(r=.218, N=23, p=.159$, one-tailed) for the simple-RT task, nor for the choice-RT task ( $r=$ $.111, N=23, p=.307$, one-tailed), suggesting that the aged group were homogeneous with respect to overall performance.

\section{Errors}

The proportion of trials in which participants failed to respond was calculated for each subject, each hand and each condition, and converted into percentages of overall missed RTs per condition. Data from four younger and five older participants, not included in the final groups of 23 and 29 participants, in whom errors exceeded $15 \%$ in any one condition, were excluded from analysis. For the simple RT task, the average percentages of missed RTs for the remaining participants were less than $1 \%$ for both age groups. For the choice RT task the younger group failed to respond to $4.06 \%$ of the stimuli presentations and the older group $6.28 \%$. Incorrect responses in the choice RT task amounted to $7.13 \%$ of the trials for the younger group and $8.08 \%$ of the trials for the older group. Trials in which participants failed to respond, and trials in which incorrect responses were made, were excluded from further analysis. There were very few catch trial errors; in the simple RT the younger group had a total of nine false alarms and the older group had a total of two. No single participant had more than one false alarm. There were no responses to catch trials for either age group in the choice RT task.

\section{Analyses}

As an initial exploration of the data, mean RTs were calculated for each of the two tasks, and separately for the combinations of hand and visual field. Participants responded to targets $210 \mathrm{~ms}$ faster, on average, in the simple RT task $(M=305 \mathrm{~ms})$ than in the choice RT task $(M=515 \mathrm{~ms})$. A repeated-measures $t$ test found this difference to be significant, $t(51)=35.804, p<.001$. As expected, based on the hand preference of the participants, responses to bilateral targets were, on average, faster with the right hand $(M=393 \mathrm{~ms})$ than with the left hand ( $M=403 \mathrm{~ms}), t(51)=$ $3.387, p=.001$. Responses with the right hand were faster to RVF stimuli (408 ms) than to LVF stimuli (421 ms). Responses with the left hand were faster to LVF stimuli (421 ms) than to RVF stimuli (424 ms).

To address the central question of the study, and to explore group differences in the RTE and the CUD, analyses of variance were applied. As previous research (Corballis, 2002; Schulte et al., 2004) has shown little evidence of significant association, within participant groups, between these two measures, correlation analyses were not employed.

\section{The RTE}

The calculated RTEs were analyzed using a three-way mixed factorial analysis of variance (ANOVA) to test the significance of 
the RTE and explore possible age/hand/task interactions. Age group (younger, older) was the between-subjects factor and hand (left, right) and task (simple RT, choice RT) were the withinsubjects factors.

The ANOVA revealed a main effect of age $F(1,50)=4.914$, $p=.031, \eta_{\mathrm{p}}^{2}=.089(M=10.29, S D=10.37$ and $M=17.45$, $S D=12.84$ for the younger and older group, respectively) (See Table 1 and Table 2 for the simple and choice task, respectively). None of the within-subjects main effects or interactions was significant. In relation to the predictions that meant that task difficulty did not significantly influence the RTE, $F(1,50)=.352, p=.556$, $\eta_{\mathrm{p}}^{2}=.007$, and as the age group by task interaction was not significant, the difference in the RTE between the younger and older group did not significantly differ as a function of task $F(1$, $50)=.003, p=.959, \eta_{\mathrm{p}}^{2}=.000$. This finding contradicted the hypothesis that age effects on the RTE would be greater in the choice task.

As can be seen from Table 1 and Table 2 both age groups showed a redundant target effect in both tasks, but the older group showed a larger RTE than the younger group for both hands and in both tasks. That the RTEs were larger for the older group was consistent with predictions. However, the prediction that ageeffects would be maximal for the choice RT task was not supported.

Having determined that the bilateral stimuli presentations led to a facilitation of reaction times, further analyses were needed in order to determine whether the observed redundant target effects exceeded that predicted by the race model inequality, implying neural coactivation.

\section{Testing the Race Model Inequality}

In order to examine whether the observed RTEs exceeded that which could be explained by the race model inequality as arising purely from statistical facilitation, cumulative distribution functions (CDFs) were created for each participant, each hand, and each task. According to the standard race model, when bilateral redundant stimuli are presented, the stimuli in the visual field ipsilateral and contralateral to the responding hand initiate separate processes, and the final response (RT) is determined by whichever process completes first. The probability (PB) that a response to bilateral stimuli will have occurred by time $t$ is as follows: $\mathrm{PB}_{\mathrm{t}}=$ $\mathrm{PL}_{\mathrm{t}}+\mathrm{PR}_{\mathrm{t}}-\left(\mathrm{PL}_{\mathrm{t}} * \mathrm{PR}_{\mathrm{t}}\right)$, where $\mathrm{PL}_{\mathrm{t}}$ is the probability of a response having occurred within time to the stimulus in the LVF, and $\mathrm{PR}_{\mathrm{t}}$ is the probability of a response having occurred to the stimulus in the RVF. This model can be tested by plotting the cumulative distributions over time of $[\mathrm{PB}-(\mathrm{PL}+\mathrm{PR}-\mathrm{PL} * \mathrm{PR})]$ (Miller, 1982; Miller, 2004). This equation assumes that the processes are independent - this assumption is relaxed in the race model inequality. The equation for the race model inequality is as follows: $\mathrm{PB}_{\mathrm{t}} \leq \mathrm{PL}_{\mathrm{t}}+\mathrm{PR}_{\mathrm{t}}$. The race model inequality can be tested by plotting the cumulative distributions over time in the following manner: [PB - (PL + PR)] (Miller, 1982; Miller, 2004). The race model inequality was chosen in this study, avoiding the need to assume independence.

In each task each participant received 180 stimulus presentations per hand (excluding catch trials); 60 in the LVF, 60 in the RVF and 60 bilateral stimuli. RTs were ranked ascending and any missed trials deleted. RTs were then assigned to 18 bins of 10 , with the first bin containing the 10 fastest RTs, the second bin the next 10 fastest RTs and so forth. The RTs in each bin were then assigned to each stimulus condition (LVF, RVF, Bilateral) and cumulative probabilities for each stimulus condition were calculated. The cumulative distribution functions for the race model inequality $[\mathrm{PB}-(\mathrm{PL}+\mathrm{PR})]$ were then calculated, thereby obtaining four race model inequality plots per participant (one for each hand and each task). The means and standard deviations $(S D)$ of the race model inequality are shown in Figure 1.

Tests of the race model inequality produced little evidence for coactivation, defined as values greater than zero (Colonius \& Diederich, 2006), although positive values were present for many individuals, particularly over the early response bins. This was true for both age groups, but only for the simple-RT task.

\section{The CUD}

The median RTs were analyzed using a higher order mixed factorial ANOVA to investigate the CUD and explore possible effects of age and task/hand/visual field interactions. Age group (younger, older) was the between-subjects factor, and task (simple, choice), hand (left, right), and visual field (LVF, RVF) withinsubjects factors. Mean and standard deviation CUDs for each age-group, hand and task, are provided in Table 1 and Table 2 for the simple and choice task, respectively. A significant CUD manifested as an interaction between hand and visual field, $F(1,50)=$ 26.658, $p<.000, \eta_{\mathrm{p}}^{2}=.348$. There were no reliable differences between the CUDs for the younger and older group, as demonstrated by the finding that the interaction between age-group, hand, and visual fields was not significant $F(1,50)=.024, p=.879$, $\eta_{\mathrm{p}}^{2}=.000$, nor was this interaction affected by the factor of task, as the interaction between age group, task, hand and visual field was not significant, $F(1,50)=.345, p=.560, \eta_{\mathrm{p}}^{2}=.007$. In the choice task both age groups showed a negative CUD for the left hand.

Table 1

Means of Median RTs, CUD, and RTE (in ms) for Simple RT Task for Both Age Groups

\begin{tabular}{|c|c|c|c|c|c|c|c|c|c|c|}
\hline & \multicolumn{5}{|c|}{ Left hand } & \multicolumn{5}{|c|}{ Right hand } \\
\hline & LVF & RVF & Bilateral & CUD & RTE & LVF & RVF & Bilateral & CUD & RTE \\
\hline Younger & $292(48)$ & $299(55)$ & $280(52)$ & $7(16)$ & $12(14)$ & $297(40)$ & 281 (39) & 270 (39) & $16(16)$ & $11(15)$ \\
\hline Older & 337 (57) & $345(60)$ & $318(57)$ & $8(24)$ & $19(16)$ & 334 (66) & 324 (59) & $306(62)$ & $10(22)$ & 17 (14) \\
\hline
\end{tabular}

Note. Standard deviations in brackets. 
Table 2

Means of Median RTs, CUD, and RTE (in ms) for Choice RT Task for Both Age Groups

\begin{tabular}{|c|c|c|c|c|c|c|c|c|c|c|}
\hline & \multicolumn{5}{|c|}{ Left hand } & \multicolumn{5}{|c|}{ Right hand } \\
\hline & LVF & RVF & Bilateral & CUD & RTE & LVF & RVF & Bilateral & CUD & RTE \\
\hline Younger & $496(54)$ & $493(48)$ & $483(51)$ & $-3(23)$ & $13(25)$ & $496(55)$ & $484(51)$ & $478(47)$ & $13(26)$ & $5(24)$ \\
\hline Older & $560(55)$ & $558(49)$ & $544(49)$ & $-2(27)$ & $17(26)$ & $558(53)$ & $544(48)$ & $527(46)$ & $14(28)$ & $17(27)$ \\
\hline
\end{tabular}

Note. Standard deviations in brackets.

\section{Discussion}

\section{The RTE}

The redundant target effect is a means of testing visuomotor interhemispheric integration. Both age groups showed a RTE in both tasks and with both hands. Consistent with predictions, the older group of participants showed a larger overall RTE than the younger group with both hands in both the simple and the choice tasks. The observation of larger RTEs with age is consistent with the assumptions of the Hemispheric Coactivation Model (Miller, 2004) that compromised or reduced interhemispheric connectivity
Simple RT, younger group, left hand

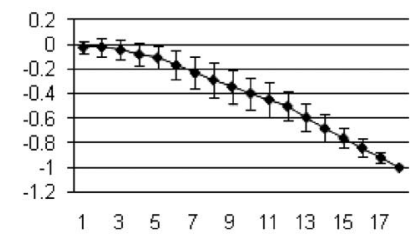

Simple RT, older group, left hand

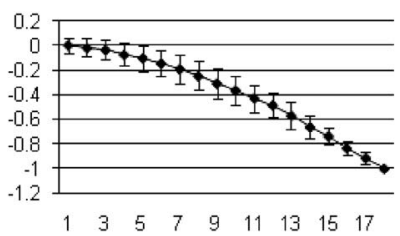

Choice RT, younger group, left hand

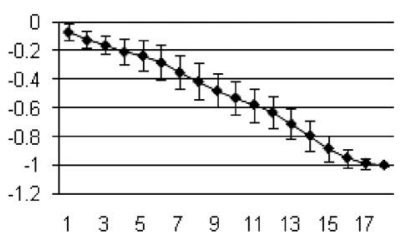

Choice RT, older group, left hand

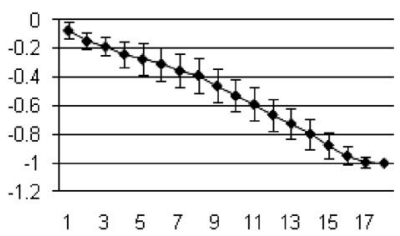

Simple RT, younger group, right hand

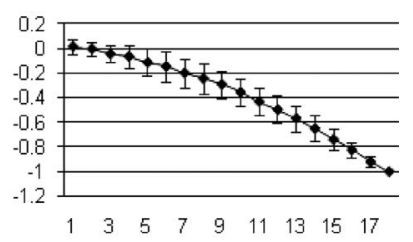

Simple RT, older group, right hand

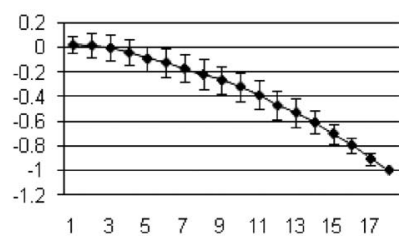

Choice RT, younger group, right hand

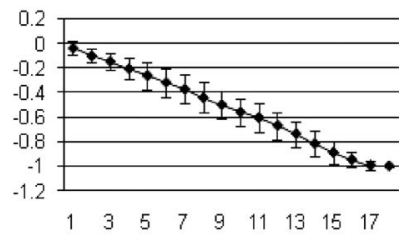

Choice RT, older group, right hand

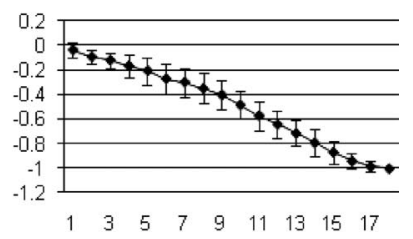

Figure 1. Means and SDs of the race model inequality $[\mathrm{PB}-(\mathrm{PL}+\mathrm{PR})]$ $(y$-axis) plotted over 18 response bins ( $x$-axis) for each combination of age group/hand/task. would result in a greater advantage of bilateral presentation, relative to unilateral presentation, and thus an increased RTE. This result adds to recent descriptions of age-related increases in the benefit from redundant modalities (Bucur, Allen et al., 2005), or dimensions (Bucur, Madden et al., 2005), which were interpreted as suggestive of attentional compensation for age-related deficits in perceptual processing. In the present paradigm, and following from the Hemispheric Coactivation Model, the presentation of bilateral redundant stimuli compensates for reduced interhemispheric connectivity in the aged brain, producing a RTE larger than that seen in young controls.

In this model, callosal transfer leads to bihemispheric processing of unilateral stimuli, resulting in similar patterns of cortical activity following unilateral or bilateral stimulation. Impaired callosal transfer reduces processing by the hemisphere ipsilateral to a unilateral stimulus, thereby reducing the similarity of cortical activity in response to unilateral or bilateral stimulation. This leads to an increase in the difference between RTs in these two conditions, and to a larger RTE. The present results suggest that age, which is associated with reduced callosal efficiency, produces a similar effect.

Although RTEs were observed for all comparisons of RTs to bilateral-redundant stimuli with RTs to unilateral stimuli, tests of the race model inequality provided little evidence for coactivation, with violations of the RMI restricted to data in early response bins from the simple RT task, and from only a subset of individuals. This stands in stark contrast to the large RTEs and race-model violations shown in studies of callosally sectioned patients (Iacoboni \& Zaidel, 2003a; Roser \& Corballis, 2002, 2003; ReuterLorenz et al., 1995), and suggests that, although age is associated with an increase in the bilateral RTE, the effect of age-related degeneration to interhemispheric tracts is markedly less than that of surgical disconnection. Nonetheless, one account, the Hemispheric Coactivation Model, can explain findings from both surgical cases and aged individuals. Our previous research including individuals with varying degrees of disconnection (Roser \& Corballis, 2002, 2003) found a continuum of bilateral RTE that paralleled the degree of hemispheric disconnection. Acallosal patients, born without a corpus callosum, showed a bilateral RTE greater than that of neurologically normal controls. Callosotomised patients, in whom the corpus callosum is cut, produced a larger bilateral RTE than did acallosal patients. Commissurotomised patients, in whom the corpus callosum and several other commissures are cut, produced a bilateral RTE larger still. The present result suggests that aged individuals, in whom interhemispheric structural connectivity is known to be degraded, lie at one end of this continuum of disconnection, a position reflected in their small, 
but significant, increase in bilateral RTE relative to that seen in young controls.

The age of our sample of older adults may explain why evidence for coactivation was minimal in the current study, in contrast to previous investigations of redundancies of modality (Bucur, Allen et al., 2005) and dimension (Bucur, Madden, et al., 2005). Bucur and colleagues employed a sample of older adults that ranged in age from 60 to $85(M=69)$ years, whereas the sample in the current study comprised adults aged 55-74 $(M=61)$. The present sample is relatively young compared to the age range across which brain structural degeneration has been demonstrated (Sullivan \& Pfefferbaum, 2006) and it is possible that age effects were mild in this group.

An alternative explanation for the disparity of evidence for coactivation between this study and previous studies of age effects on the RTE is that age-related decline in attentional capacity may manifest as an enhanced RTE in paradigms employing redundancy of modality or dimension more readily than do the subtle effects of age upon callosal connectivity in the bilateral redundancy paradigm. Nonetheless, the most important finding, that of an agerelated increase in RTE, is common to both paradigms.

The lack of strong violation of the race model inequality is congruent with previous studies of neurologically normal individuals (Corballis, 1998; Corballis, 2002; Roser \& Corballis, 2002; Barr \& Corballis, 2003; Corballis, Hamm, Barnett, \& Corballis, 2002; Murray, Foxe, Higgins, Javitt, \& Schroeder, 2001; ReuterLorenz et al., 1995) and suggests that much of the RTE effect can be accounted for by statistical facilitation. Despite these negative results there is still good reason to believe that neural summation often occurs in response to redundant stimuli. For example, many studies have reported enhanced RTEs and violations of Miller's (1982) RMI in neurologically intact individuals, thus supporting neural summation, or coactivation mechanisms (Miniussi et al., 1998; Roser \& Corballis, 2003; Savazzi \& Marzi, 2002; CavinaPratesi, Bricolo, Prior, \& Marzi, 2001; Iacoboni \& Zaidel, 2003b). Furthermore, the RMI provides a conservative test of the race model; the inequality necessarily is satisfied for large values of $t$ (later response bins), because the left side approaches an upper limit of one, whereas the right side approaches an upper limit of two (Miller \& Van Nes, 2007). This conservatism argues against finding a violation of the race model. Taken together, this evidence suggests that neural coactivation of responses to redundant stimuli is a common mode of processing in the brain which is fleetingly unmasked by neurological disconnection or cognitive degeneration.

It was predicted that the effect of age on the RTE would be more pronounced in the choice RT task, given that it likely involves greater reliance on frontal regions which have been shown to be particularly vulnerable to age related declines in microstructural integrity. This prediction was not supported as the effects of age on the RTE were comparable between tasks. A possible explanation for this observation is that the effect of increased task complexity on bihemispheric processing in the aged brain may have mitigated redundancy gain arising from any degeneration of frontal interhemispheric connectivity. To understand this conjecture it is first necessary to note that behavioral studies suggest that both younger and older adults benefit from bihemispheric processing at high levels of task complexity, and that bihemispheric processing may be beneficial to older adults at lower levels of task complexity than it is to young participants (Banich, 1998; Reuter-Lorenz et al., 1999). Furthermore, neuroimaging studies have demonstrated that under difficult task conditions, older adults show greater bilateral activation, particular in frontal areas, compared to younger adults (Cabeza, 2002; Park \& Reuter-Lorenz, 2009). In addition, for a number of motor tasks, older adults have been shown to recruit additional cortical as well as subcortical regions that are not activated in younger adults (Carp et al., 2010; Heuninckx et al., 2008; Mattay et al., 2002; Seidler et al., 2010), and increasing the task complexity in motor tasks have been found to lead to an increase in the recruitment of additional brain areas, particularly the ipsilateral M1 (Seidler, Noll, \& Thiers, 2004). Therefore, in the choice RT task in the current experiment, two effects of age may have operated in opposition. First, increased reliance on frontal processing, subserved by frontal white matter tracts highly vulnerable to age-related degeneration, may have acted to increase age effects on the bilateral RTE, relative to the simple RT task, as routes crucial for hemispheric transfer were possibly compromised. This follows from the Hemispheric Coactivation Model in which bihemispheric processing occurs in response to unilateral stimulation, except in the disconnected brain. Second, conditions of increased task difficulty may have led to a general increase in bihemispheric processing, particularly in the aged, as seen in previous neuroimaging studies. Increased bihemispheric activation in the aged group would reduce the difference in brain activity between conditions of single or redundant stimulation and act against the bilateral RTE. Thus the increased task demands required by the choice task in this study may have led to a reduction in the differences of brain activity between the two age groups, resulting in no interaction of age group and task in the size of the RTE. Teasing apart possibly opposed processes, and their neural substrates, will likely depend on neuroimaging studies of the bilateral RTE in which patterns of functional activation are observed in young and old participants as task demands are manipulated.

\section{The CUD}

A second line of investigations concerned interhemispheric visuomotor transfer, as assessed by the crossed-uncrosseddifference. The prediction that older participants were expected to show larger CUDs than the younger participants, and that those age differences would be more pronounced in the choice task given that the choice task likely involves greater reliance on frontal regions, was not supported. A small number of studies have investigated the CUD in healthy older individuals, with equivocal results (Hoptman et al., 1996; Jeeves \& Moes, 1996; ReuterLorenz \& Stanczak, 2000; Schulte et al., 2004), Furthermore, in line with previous evidence (Saron \& Davidson, 1989; Davidson, Leslie, \& Saron, 1990), both age groups showed a negative CUD for the left hand in the choice task which contradicts the basic tenet of the CUD paradigm. Several past results are germane to interpretation of these current data. Electrophysiological studies have demonstrated that uni-manual responses involves bilateral frontal, central, and occipital activations (Saron, 1999), and MEG studies have shown that unimanual index finger movements involve bilateral motor cortex activations (Babiloni et al., 1999). Furthermore, the CUD has been found to vary significantly within individuals and with different types of experimental conditions, and 
furthermore require numerous trials (thousands) for a stable measurement (Iacoboni \& Zaidel, 2000). Together these results strongly suggest that the simple anatomical model on which the CUD is based may be invalid (for a review see Braun, Achim, \& Laroque, 2003). It has been suggested that subtracting the RT in uncrossed conditions from the RT in crossed conditions may in fact "contrast one form of interhemispheric interaction with another, rather than the presence versus absence of callosal mediation" (Saron, Foxe, Schroeder, \& Vaughan, 2004, p. 393). There are numerous interhemispheric channels involved even in these simple tasks; visual transfer via the splenium, motor transfer via the midbody and the genu connecting frontal areas involved in motor control and response selection. Ageing could affect all of these processes, but further research that combines behavioral measures with electroencephalography measures and DTI assessing microstructural integrity is necessary in order to clarify both individual variability and how this may be related to functional variations in visuomotor interhemispheric transfer.

\section{Conclusion}

The present study contrasted unilateral versus redundant bilateral stimulation, enabling the investigation of visuomotor interhemispheric integration, and thus provides novel evidence that the magnitude of the bilateral RTE is increased in healthy older individuals. This increased RTE with age is consistent with Miller's (2004) hemispheric coactivation model and evidence for agerelated degeneration of cerebral white matter microstructural integrity. Contrary to predictions the RTE was not larger in the choice task than the simple RT task.

Minor disruptions to callosal integrity, due to normal ageing, may have functional ramifications, and may be the underlying cause of the increased RTEs observed for the older group. Further investigations incorporating functional MRI indices of cortical processing as well as DTI measures assessing microstructural integrity are needed to further our understanding of the relationship between individual differences in neural activation patterns and brain connectivity, and the implications for behavior. Research investigating these phenomena in healthy older individuals is lacking, but is important for the understanding of fundamental visuomotor behavior.

\section{References}

Allen, P. A., Groth, K. E., Weber, T. A., \& Madden, D. J. (1993). Influence of response selection and noise similarity on age differences in the redundancy gain. Journal of Gerontology: Psychological Sciences, 48, 189-198.

Allen, P. A., Madden, D. J., Groth, K. E., \& Crozier, L. C. (1992). Impact of age, redundancy, and perceptual noise on visual search. Journal of Gerontology, 47, 69-74.

Allen, P. A., Weber, T. A., \& Madden, D. J. (1994). Adult age differences in attention: Filtering or selection? Journal of Gerontology, 49, 213222.

Babiloni, C., Carducci, F., Pizzella, V., Indovina, I., Romani, G. L., Rossini, P. M., . . Babiloni, F. (1999). Bilateral neuromagnetic activation of human primary sensorimotor cortex in preparation and execution of unilateral voluntary finger movements. Brain Research, 827, 234236.

Banich, M. (1998). The missing link: The role of interhemispheric interaction in attentional processing. Brain and Cognition, 36, 128-157.
Barr, M. S., \& Corballis, M. C. (2003). Redundancy gain in the acallosal brain. Neuropsychology, 17, 213.

Bennett, I. J., Madden, D. J., Vaidya, C. J., Howard, D. V., \& Howard, J. H. (2010). Age-related differences in multiple measures of white matter integrity: A diffusion tensor imagning study of healthy aging. Human Brain Mapping, 31, 379-390.

Braun, C. M., Achim, A., \& Larocque, C. (2003). The evolution of the concept of interhemispheric relay time. In E. Zaidel \& M. Iacoboni (Eds.), The parallel brain - the cognitive neuroscience of the corpus callosum. Cambridge, Massachusetts: The MIT Press.

Bucur, B., Allen, P. A., Sanders, R. E., Ruthruff, E., \& Murphy, M. D. (2005). Redundancy gain and coactivation in bimodal detection: Evidence for the preservation of coactive processing in older adults. Journal of Gerontology, 60, 279-282.

Bucur, B., Madden, D. J., \& Allen, P. A. (2005). Age-related differences in the processing of redundant visual dimensions. Psychology and $\mathrm{Ag}$ ing, 20, 435-446.

Burzynska, A. Z., Preuschhof, C., Bäckman, L., Nyberg, L., Lindenberger, U., \& Heekeren, H. R. (2010). Age-related differences in white matter microstructure: Region-specific patterns of diffusivity. Neuroimage, 49, 2104-2112.

Cabeza, R. (2002). Hemispheric asymmetry reduction in older adults: The HAROLD model. Psychology and Aging, 17, 85-100.

Cabeza, R., Anderson, N. D., Locantore, J. K., \& McIntosh, A. R. (2002). Aging gracefully: Compensatory brain activity in high-performing older adults. NeuroImage, 17, 1394-1402.

Cámara, E., Bodammer, N., Rodrigues-Fornells, A., \& Tempelmann, C. (2007). Age-related water diffusion changes in human brain: A voxelbased approach. NeuroImage, 34, 1588-1599.

Carp, J., Park, J., Polk, T. A., \& Park, D. C. (2010). Age differences in neural distinctiveness revealed by multi-voxel pattern analysis. Neuroimage. doi;10.1016/j.neuroimage.2010.04.267

Cavina-Pratesi, C., Bricolo, E., Prior, M., \& Marzi, C. A. (2001). Redundancy gain in the stop-signal paradigm: Implications for the locus of coactivation in simple reaction time. Journal of Experimental Psychology: Human Perception and Performance, 27, 932-941.

Chanraud, S., Zahr, N., Sullivan, E. V., \& Pfefferbaum, A. (2010). MR diffusion tensor imaging: A window into white matter integrity of the working brain. Neuropsychology Review, 20, 209-225.

Colonius, H., \& Diederich, A. (2006). The race model inequality: Interpreting a geometric measure of the amount of violation. Psychological Review, 113, 148-154.

Corballis, M. C. (1998). Interhemispheric neural summation in the absence of the corpus callosum. Brain, 121, 101-113.

Corballis, M. C. (2002). Hemispheric interactions in simple reaction time. Neuropsychologia, 40, 423-434.

Corballis, M. C., Corballis, P. M., \& Fabri, M. (2003). Redundancy gain in simple reaction time following partial and complete callosotomy. Neuropsychologia, 42, 71-81.

Corballis, M. C., Hamm, J. P., Barnett, K. J., \& Corballis, P. M. (2002). Paradoxical interhemispheric summation in the split brain. Journal of Cognitive Neuroscience, 14, 1151-1157.

Davidson, R. J., Leslie, S. C., \& Saron, C. (1990). Reaction time measures of interhemispheric transfer time in reading disabled and normal children. Neuropsychologia, 28, 471-485.

Dickerson, B. C., \& Sperling, R. A. (2008). Functional abnormalities of the medial temporal lobe memory systems in mild cognitive impairment and Alzheimer's disease: Insights from functional MRI studies. Neuropsychologia, 46, 1624-1635.

Folstein, M. F., Folstein, S. E., \& McHugh, P. R. (1975). Mini-Mental State Examination. A practical method for grading the cognitive state of patients for the clinician. Journal of Psychiatric Research, 12, 189-198. Good, C. D., Johnsrude, I. S., Ashburner, J., Henson, R. N. A., Friston, 
K. J., \& Frackowiak, R. S. J. (2001). A voxel-based morphometric study of ageing in 465 normal adult human brains. Neuroimage, 14, 21-36.

Head, D., Buckner, R. L., Shimony, J. S., Williams, L. E., Akbudak. E., Conturo, T. E., ... Snyder, A. Z. (2004). Differential vulnerability of anterior white matter in nondemented aging with minimal acceleration in dementia of the Alzheimer type: Evidence from diffusion tensor imaging. Cerebral Cortex, 14, 410-423.

Heuninckx, S., Wenderoth, N., \& Swinnen, S. P. (2008). Systems neuroplasticity in the aging brain: Recruiting additional neural resources for successful motor performance in elderly persons. The Journal of Neuroscience, 28, 91-99.

Hoptman, M. J., Davidson, R. J., Gudmundsson, A., Schreiber, R. T., \& Ershler, W. B. (1996). Age differences in visual evoked potential estimates on interhemispheric transfer. Neuropsychology, 10, 263-271.

Iacoboni, M., Ptito, A., Weekes, N. Y., \& Zaidel, E. (2000). Parallel visuomotor processing in the split brain: Cortico-subcortical interactions. Brain, 123, 759-769.

Iacoboni, M., \& Zaidel, E. (2000). Crossed-uncrossed difference in simple reaction times to lateralized flashes: Between- and within-subjects variability. Neuropsychologia, 38, 535-541.

Iacoboni, M., \& Zaidel, E. (2003a). Stable and variable aspects of callosal channels: Lessons from partial disconnection. In E. Zaidel \& M. Iacoboni (Eds.), The Parallel Brain - The Cognitive Neuroscience of the Corpus Callosum. Massachusetts: The MIT Press.

Iacoboni, M., \& Zaidel, E. (2003b). Interhemispheric visuo-motor integration in humans: The effect of redundant targets. European Journal of Neuroscience, 17, 1981-1986.

Jeeves, M. A., \& Moes, P. (1996). Interhemispheric transfer time differences related to aging and gender. Neuropsychologia, 34, 627-636.

Langan, J., Peltier, S., Bo., J., Fling, B. W., Welsh, R. C., \& Seidler, R. D. (2010). Functional implications of age differences in motor system connectivity. Frontiers in Systems Neuroscience, 4, 1-11. doi:10.3389/ fnsys.2010.00017

Mattay, V. S., Fera, F., Tessitore, A., Hariri, A. R., Das, S., Callicot, J. H., . . Weinberger, D. S. (2002). Neurophysiological correlates of age-related changes in human motor function. Neurology, 58, 630-635.

Miller, J. (1982). Divided attention: Evidence for coactivation with redundant signals. Cognitive Psychology, 14, 247-279.

Miller, J., \& Van Nes, F. (2007). Effects of response task and accessory stimuli on redundancy gain: Tests of the hemispheric coactivation model. Journal of Experimental Psychology, 33, 829-844.

Miller, J. O. (2004). Exaggerated redundancy gain in the split brain: A hemispheric coactivation account. Cognitive Psychology, 49, 118-154.

Miniussi, C., Girelli, M., \& Marzi, C. (1998). Neural site of the redundant target effect: Electrophysiological evidence. Journal of Cognitive Neuroscience, 10, 216-230.

Murray, M. M., Foxe, J. J., Higgins, B. A., Javitt, D. C., \& Schroeder, C. E. (2001). Visuo-spatial neural response interactions in early cortical processing during a simple reaction time task: A high-density electrical mapping study. Neuropsychologia, 39, 828-844.

Oldfield, R. C. (1971). The assessment and analysis of handedness: The Edinburgh Handedness Inventory. Neuropsychologia, 9, 97-113.

Ota, M., Obata, T., Akine, Y., Ito, H., Ikehira, H., Asada, T., \& Suhara, T. (2006). Age-related degeneration of corpus callosum measured with diffusion tensor imaging. NeuroImage, 31, 1445-1452.

Ouimet, C., Jolicoeur, P., Miller, J., Ptito, A., Paggi, A., Foschi, N., ... Lassonde, M. (2009). Sensory and motor involvement in the enhanced redundant target effect: A study comparing anterior- and totally split-brain individuals. Neuropsychologia, 47, 684-692.

Park, D. C., \& Reuter-Lorenz, P. (2009). The adaptive brain: Aging and neurocognitive scaffolding. The Annual Review of Psychology, 60, 173196.

Raab, D. (1962). Statistical facilitation of simple reaction times. Transactions of the New York Academy of Sciences, 24, 574-590.
Raz, N., Gunning, F. M., Head, D., Dupuis, J. H., McQuain, J., Briggs, S. D., ... Acker, J. D. (1997). Selective aging of the human cerebral cortex observed in vivo: Differential vulnerability of the prefrontal gray matter. Cerebral Cortex, 7, 268-282.

Raz, N., Lindenberger, U., Rodrigue, K. M., Kennedy, K. M., Head, D., Williamson, A., . . . Acker, J. D. (2005). Regional brain changes in aging healthy adults: General trends, individual differences and modifiers. Cerebral Cortex, 15, 1676-1689.

Raz, N., \& Rodrigue, K. M. (2006). Differential aging of the brain: Patterns, cognitive correlates and modifiers. Neuroscience and Biobehavioural Reviews, 30, 730-748.

Reuter-Lorenz, P. A., \& Mikels, J. A. (2005). A split-brain model of Alzheimer's disease? Behavioural evidence for comparable intra and interhemispheric decline. Neuropsychologia, 43, 1307-1317.

Reuter-Lorenz, P. A., Nozawa, G., Gazzaniga, M. S., \& Hughes, H. C. (1995). The fate of neglected targets in the callosotomized brain: A chronometric analysis. Journal of Experimental Psychology, 21, 211230.

Reuter-Lorenz, P. A., \& Stanczak, L. (2000). Differential effects of aging on the functions of the corpus callosum. Developmental Neuropsychology, 18, 113-137.

Reuter-Lorenz, P. A., Stanczak, L., \& Miller, A. C. (1999). Neural recruitment and cognitive aging: Two hemispheres are better than one, especially as you age. Psychological Science, 10, 494-500.

Roser, M., \& Corballis, M. C. (2002). Interhemispheric neural summation in the split brain with symmetrical and asymmetrical displays. Neuropsychologia, 40, 1300-1312.

Roser, M., \& Corballis, M. C. (2003). Interhemispheric neural summation in the split brain: Effects of stimulus colour and task. Neuropsychologia, $41,830-846$.

Roser, M. E., Corballis, M. C., Jansari, A., Fulford, J., Benattayallah, A, \& Adams, W. M. (in press). Bilateral redundancy gain and callosal integrity in a man with callosal lipoma: A diffusion-tensor imaging study. Neurocase.

Salat, D. H., Tuch, D. S., Greve, D. N., Kouwe, A. J. W., Hevelone, N. D., Zaleta... Dale, A. M. (2005). Age-related alterations in white matter microstructure measured by diffusion tensor imaging. Neurobiology of Aging, 26, 1215-1227.

Saron, C. D. (1999). Spatiotemporal electrophysiology of intra- and interhemispheric visuomotor integration: Relations with behaviour. Doctoral dissertation, Albert Einstein College of Medicine, Bronx, New York.

Saron, C. D., \& Davidson, R. J. (1989). Visual evoked potential measures of interhemispheric transfer time in humans. Behavioral Neuroscience, 103, 1115-1138.

Saron, C. D., Foxe, J. J., Schroeder, C. E., \& Vaughan, H. G. (2004) Complexities of interhemispheric communication in sensorimotor tasks revealed by high-density event-related potential mapping. In K. Hugdahl, \& R. J. Davidson (Eds.), The asymmetrical brain, Cambridge, Massachusetts: The MIT Press.

Savazzi, S., \& Marzi, C. A. (2002). Speeding up reaction time with invisible stimuli. Current Biology, 12, 403-407.

Schulte, T., Chen, S. H. A., Müller-Oehring, E. M., Adalsteinsson, E., Pfefferbaum, A., \& Sullivan, E. V. (2006). fMRI evidence for individual differences in premotor modulation of extrastriatal visual-perceptual processing of redundant targets. NeuroImage, 30, 973-982.

Schulte, T., Pfefferbarum, A., \& Sullivan, E. V. (2004). Parallel interhemispheric processing in aging and alcoholism: Relation to corpus callosum size. Neuropsychologia, 42, 257-271.

Schulte, T., Sullivan, E. V., Müller-Oehring, E. M., Adalsteinsson, E., \& Pfefferbaum, A. (2005). Corpus callosal microstructural integrity influences interhemispheric processing: A diffusion tensor imaging study. Cerebral Cortex, 15, 1384-1392.

Seidler, R. D., Bernard, J. A., Burutolu, T. B., Fling, B. W., Gordon, M. T., Gwin, J. T., . . L Lipps, D. B. (2010). Motor Control and aging: Linnks to 
age-related brain structural, functional, and biochemical effects. Neuroscience and Biobehavioural Reviews, 34, 721-733.

Seidler, R. D., Noll, D. C., \& Thiers, G. (2004). Feedforward and feedback processes in motor control. Neuroimage, 22, 1775-1783.

Simon, J. R. (1969). Reaction towards the source of stimulation. Journal of Experimental Psychology: Human Perception and Performance, 81, 174-176.

Sullivan, E. V., \& Pfefferbaum, A. (2006). Diffusion tensor imaging and aging. Neuroscience and Biobehavioural Reviews, 30, 749-761.

Sullivan, E. V., Pfefferbaum, A., Adalsteinsson, E., Swan, G. E., \& Carmelli, D. (2002). Differential rates of regional brain change in callosal and ventricular size: A 4-year longitudinal MRI study of elderly men. Cerebral Cortex, 12, 438-445.
Sullivan, E. V., Rohlfing, T., \& Pfefferbaum, A. (2010). Quantitative fiber tracking of lateral and interhemispheric white matter systems in normal aging: Relations to timed performance. Neurobiology of Aging, 31, 464-481.

Zaidel, E., \& Iacoboni, M. (2003). Introduction: Poffenberger's simple reaction time paradigm for measuring interhemispheric transfer time. In E. Zaidel \& M. Iacoboni (Eds.), The parallel brain - the cognitive neuroscience of the corpus callosum. Cambridge Massachusetts: The MIT Press.

Received November 1, 2010 Revision received June 7, 2011

Accepted June 14, 2011

\section{ORDER FORM}

Start my 2012 subscription to Psychology and Aging ISSN: 0882-7974

$\$ 77.00$

APA MEMBER/AFFILIATE

$\$ 188.00$ INDIVIDUAL NONMEMBER

\section{$\$ 597.00$ INSTITUTION}

In DC and MD add $6 \%$ sales tax

TOTAL AMOUNT DUE

\section{$\$$}

Subscription orders must be prepaid. Subscriptions are on a calendar year basis only. Allow 4-6 weeks for delivery of the first issue. Call for international subscription rates.

\section{SEND THIS ORDER FORM TO}

American Psychological Association

Subscriptions

750 First Street, NE

AMERICAN

PSYCHOLOGICAL

ASSOCIATION
Call 800-374-2721 or 202-336-5600

Fax 202-336-5568 :TDD/TTY 202-336-6123

For subscription information,

e-mail: subscriptions@apa.org $\square$ Check enclosed (make payable to APA)

Charge my: $\square$ Visa $\square$ MasterCard $\square$ American Express

Cardholder Name

Card No. _ Exp. Date

Signature (Required for Charge)

\section{Billing Address}

Street

City State Zip

Daytime Phone

E-mail

Mail To

Name

Address

City $\_$State Zip _ _

APA Member \# 